\title{
Avoidance of Facial Nerve Injury in Lateral Skull Base Surgery
}

\author{
Michael E Sughrue \\ Department of Neurological Surgery, University of Oklahoma, Oklahoma City, USA
}

Correspondence: Michael E Sughrue, Comprehensive Brain Tumor Center, Department of Neurological Surgery, University of Oklahoma, 1000 North Lincoln Blvd, Suite 400, Oklahoma City, OK 73104-5023, USA, Phone: (405) 271-4912 e-mail: mes261@ columbia.edu

\begin{abstract}
In the radiosurgery era, the treatment strategies for vestibular schwannoma have changed at most centers. This new paradigm holds that rational CPA tumor therapy requires balancing often competing goals of therapy, and the associated risks and benefits of different therapies or combination of therapies. The present review discusses this new paradigm and its specific implications for CPA tumor surgery. Inevitably, such a review will focus largely on minimizing facial nerve morbidity, as this is both the most modifiable risk, and the risk that is most reduced with the use of adjuvant therapies, such as stereotactic radiosurgery (like Gamma knife). The facts about facial nerve preservation in CPA tumor surgery will be reviewed, and methods for avoiding facial nerve morbidity will be discussed.
\end{abstract}

Keywords: Vestibular schwannoma, Acoustic neuroma, Microsurgery, Facial nerve.

\section{INTRODUCTION}

A dvancement in the surgical treatment of cerebellopontine angle (CPA) tumors has been driven to its current state by at least three major paradigm shifting observations. The first came in the Cushing era, when it was realized that these tumors were too intimately related to the brainstem to be bluntly finger dissected, an observation that made surgical resection of these tumors a sane option, as the mortality rates had previously exceeded $90 \% .1,2$ The second revolution came with the introduction of the operating microscope, and the development of microsurgical techniques for dissection and preservation of the facial nerve and hearing in many cases. ${ }^{3-5}$ Finally, the introduction of skull base surgical paradigms, namely improved understanding of microsurgical anatomy, and improved surgical approaches which minimize brain retraction, have improved outcomes and provided better surgical options and reduced morbidity to rates which greatly exceed those in previous decades. ${ }^{4,6}$

I would propose that present day CPA tumor surgery is in need of a fourth paradigm shift, a shift of philosophy aw ay from single surgery monotherapy, towards a paradigm of decision-making and combined therapy. This new paradigm holds that rational CPA tumor therapy requires balancing often competing goals of therapy, and the associated risks and benefits of different therapies or combination of therapies.

The present review discusses this new paradigm and its specific implications for CPA tumor surgery. Inevitably, such a review will focus largely on minimizing facial nerve morbidity, as this is both the most modifiable risk, and the risk that is most reduced with the use of adjuvant therapies, such as stereotactic radiosurgery (like Gamma knife). ${ }^{7,8}$ The facts about facial nerve preservation in CPA tumor surgery will be reviewed, and methods for avoiding facial nerve morbidity will be discussed.

\section{Decision-Making in CPA Tumor Surgery}

For many years, aggressive surgery with the goal of gross total resection was the only reasonable option for the vast majority of patients with CPA tumors. Most tumors were diagnosed in their later stages, when tumors were large and symptomatic, good adjuvant therapies were unavailable or nonconformal, and advanced imaging was largely unavailable to closely follow residual or recurrent disease. Further, given that these tumors are largely histologically benign, slow growing lesions, meaningful outcome data for patients treated with different modalities followed over long periods of time have only recently become available, ${ }^{7,9}$ and are largely class 2 or 3 data.

As these data have become increasingly available from a large number of centers, several observations have been repeatedly demonstrated. The first is that radiosurgical and radiotherapy treatments w ork for appropriately sized tumors, they provide excellent long-term tumor control with low rates (nearly $0 \%$ using current radiosurgical dosing and targeting) of facial nerve weakness and excellent morbidity profiles. ${ }^{8,10-12}$ Further, subtotally resected vestibular schwannomas do not al ways regrow, even without adjuvant treatment, and can be followed for many years with good results. ${ }^{11,12}$ Taken together these provide an important 
al ternative to aggressive surgical resection. In other words, it is not necessary to cure the patient with surgery to achieve a successful outcome, as a good backup plan exists.

The existence of such viable alternative begs the question of whether or not it is ever wise to attempt surgical cure in these cases. This is an exceedingly complex question which requires consideration of a number of often competing goals, and the optimal balance differs among patients. D espite this, a few simplifying generalizations of the existing data can be made. First, despite the absence of well-controlled head to head comparison studies, it is reasonable to conclude that rates of tumor control for appropriately small tumors, defined as freedom from significant persistent tumor growth, are relatively similar between patients treated with surgery or radiosurgery al one. ${ }^{12}$ It should be noted that the several decades long follow-up, which is relevant for younger patients, does not exist for radiosurgery series, and thus it is important to counsel these patients that we really do not know what the best long-term solution is for them. ${ }^{12}$ Further, while radiosurgery does not seem to increase the risk of malignancy over that expected in an untreated population over the first decade following treatment, ${ }^{13}$ we still lack decades of experience with these treatments to definitively conclude that radiosurgery does not cause a significant increase in the incidence of cancer in these patients.

Where these treatments differ most is in their impact on facial nerve function. While surgical resection of small vestibular schwannomas can achieve good facial nerve outcomes in experienced hands, facial nerve palsy is rare with low dose ( $12.5 \mathrm{~Gy}$ ) radiosurgery, and it is hard to beat these results with surgery of any type, and radiosurgery is probably becoming the principle treatment for these small tumors at most centers. ${ }^{7}$ This is not to say that it is the correct treatment for all patients, how ever, in most cases it probably provides the best chance of preventing tumor growth while minimizing the risk to the facial nerve.

\section{Facial Nerve Preservation in Large Vestibular Schwannoma}

While radiosurgery is a good treatment for smaller tumors, larger tumors by necessity are treated with surgery, and in doing so, these patients are subjected to some risk of facial nerve injury. A ny honest assessment of the collective results of surgical treatment of these tumors by experienced surgeons treating these tumors with the goal of gross total resection and surgical cure is sobering. Permanent facial nerve weakness is common, and House-B rackmann grade 1 or 2 function is achieved in only 60 to $70 \%$ of these cases. $^{5,12}$ These results have led many, the author included, to question the wisdom of such an approach to a largely benign, slow growing tumor.
It is important to note that unlike radiosurgery, which is largely an all-or-none treatment modality, surgery is a malleable treatment, in that intraoperative decisions can alter the type and extent of surgical treatment provided. In such a surgical paradigm, patients undergoing surgery for small tumors are not all approached with the goal of total tumor removal, but are selectively subtotally resected, removing safe parts of the tumor, and leaving small remnants behind in surgically dangerous areas, or in other words, avoiding resection of tumor portions where facial nerve injury is likely to occur, and resecting the rest. It is important to note that such a resection is not simply a debulking, but rather represents a conscious effort to remove as much tumor as possible, stopping just before the facial nerve is put at risk. The goal in this strategy is to debulk the tumor, to devascularize it as much as possible and to leave behind a good radiosurgery target.

Does such a strategy prevent facial nerve injury? It is difficult to say. A necdotally, it seems to be uncommon in patients undergoing a true targeted subtotal resection. The existing data, however, have yet to support improved facial nerve outcomes in subtotally resected tumors, which seems counter-intuitive. ${ }^{3-6,9,11,12,14-17}$ Given that these studies did not manage these patients with the plan of stopping prior to complete tumor removal, one possible explanation for this is that when a surgeon approaches the case with the goal of taking the whole tumor out, and stops only when the facial nerve seems at dire risk or when facial nerve EM $G$ begins to show excessive spontaneous activity, by the time the decision is made to stop, then it is too late and the facial nerve may have already experienced traction or vascular injury. ${ }^{12}$ It seems reasonable to hypothesize that stopping earlier might avoid facial nerve injury in many large cases.

\section{How to Perform Targeted Subtotal Resection}

Paradoxically, subtotally resecting vestibular schwannomas can be more challenging to do well than total removal. It is difficult in many cases to know precisely when to stop. There are fewer anatomic clues to gauge the extent of resection, and it can be hard to determine the volume of residual tumor, especially when the aim is to avoid dissecting or even seeing the cisternal portion of the facial nerve. In many respects, this is analogous to a stunt pilot flying as close to the tree line as possible without hitting it.

While there are no data and only tentative experience with targeted subtotal resection to guide what portions of tumor are safe to remove, and which are best left al one, the following discussion provides a tentative strategy for performing this type of resection based on experiences and insights of myself and others. In the author's opinion, vestibular schwannomas are best thought of as five separate tumors, and that intraoperative decision making for these 

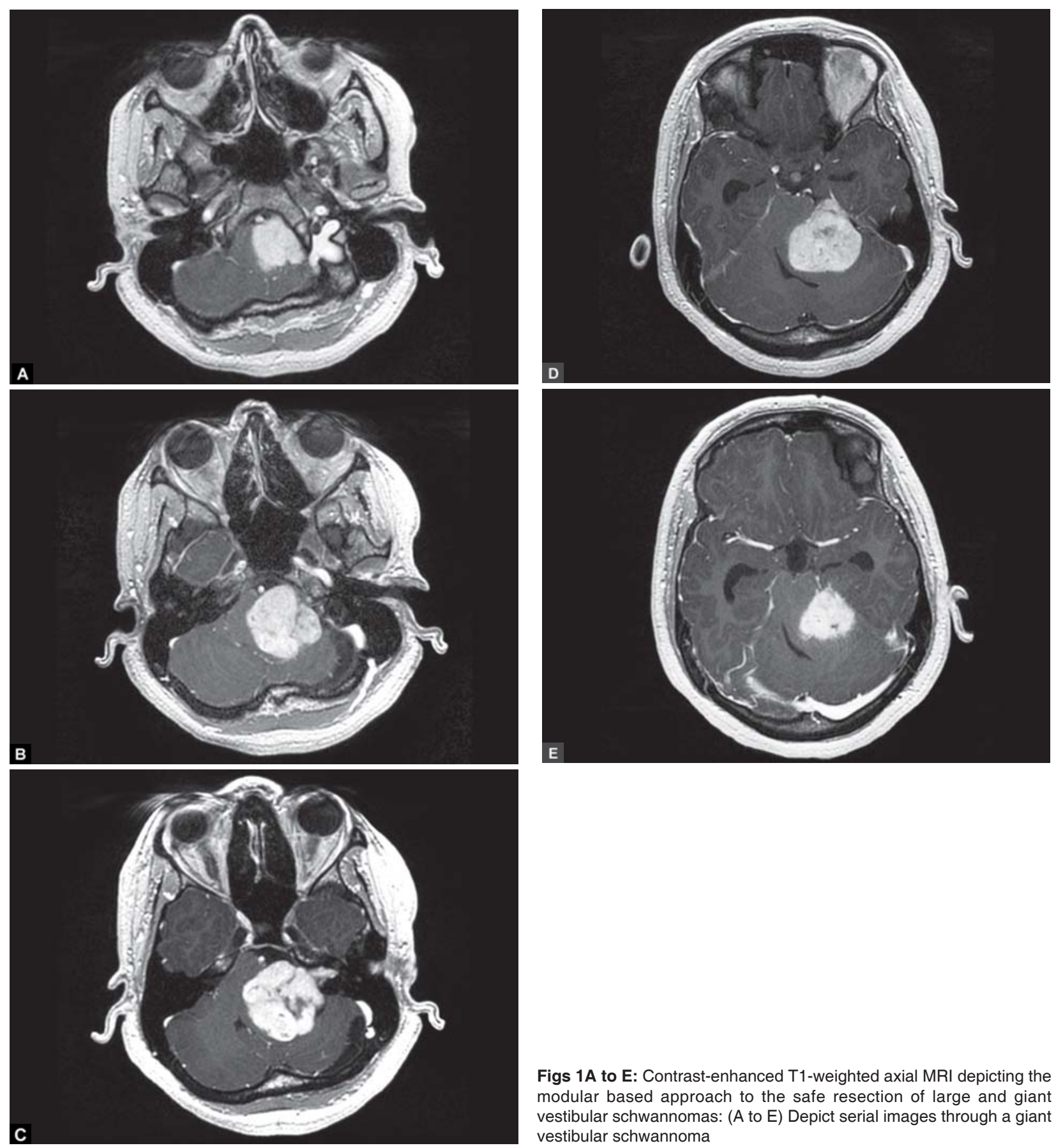

Figs 1A to E: Contrast-enhanced T1-weighted axial MRI depicting the modular based approach to the safe resection of large and giant vestibular schwannomas: (A to E) Depict serial images through a giant vestibular schwannoma

cases comprises a series of decisions made on each of these first parts. These portions are defined in Figures $1 A$ to $E$.

\section{The Cerebellar Portion}

This is typically the first portion encountered (Fig. 1F), and in most cases, this is the safest and most straightforward part of the resection. It is al so the portion when the greatest volumetric resection of the tumor occurs, and thus this resection should be maximized in the majority of cases. It is important to check the lateral, inferior and superior portions of the capsule for facial nerve fibers frequently, as in some cases, the facial nerve can be located along these surfaces, and the identification of an unfavorable intracisternal course (i.e. over the posterior surface of the tumor) of the facial nerve generally reduces the enthusiasm for aggressive resection, and at least changes the trajectory of tumor resection. Additionally, cystic tumors provide a unique challenge as in some cases, facial nerve fibers can run through the septations, necessitating repeated stimulation of these septations to ensure the absence of the facial nerve fibers prior to debulking these septations. ${ }^{18}$ 


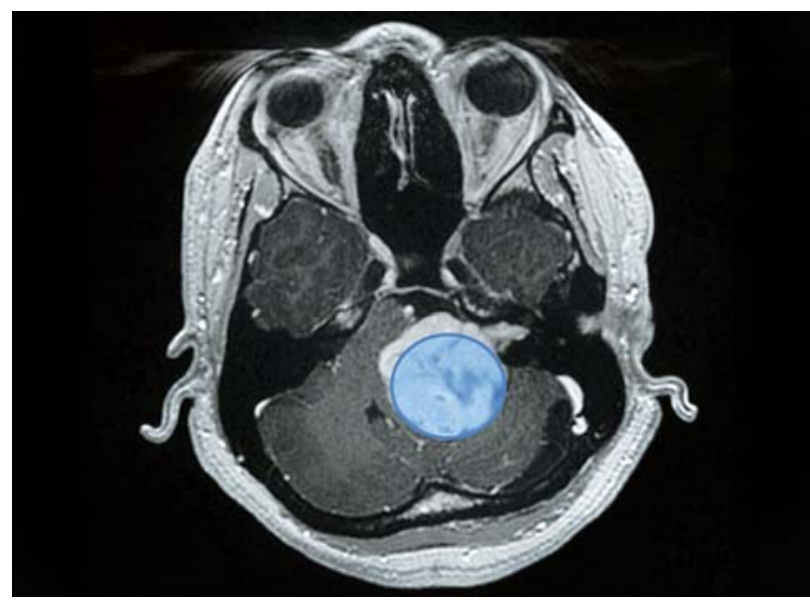

Fig. 1F: Demonstrates the section through the IAC with the cerebellar portion of the tumor outlined in blue

\section{The Brainstem Portion}

While theoretical ly el evating the tumor off of the brainstem (Fig. 1G) confers the most consequential risk to the patient during these cases, in practice, this is often less challenging than expected. Reducing the brainstem portion of the tumor can be helpful to approximate the relative location of cisternal portion of the facial nerve and thus to obtain a more compl ete resection (see bel ow). As well, by separating the tumor from the brainstem makes this tumor a better radiosurgery target. These tumors often maintain a good plane with the brainstem, and after developing a plane with the anterior cerebellar face, it is frequently straightforward to free up this face of the tumor and debulk it. In cases when this plane is not straightforward due to fibrous adhesions, it is probably not worth the risk of trying to free up this small tumor remnant. While image guidance is generally not helpful in most vestibular schwannoma surgery, it can be used to determine the approximate width of the residual fragment against the brainstem, when this fragment has unfavorable anatomy.

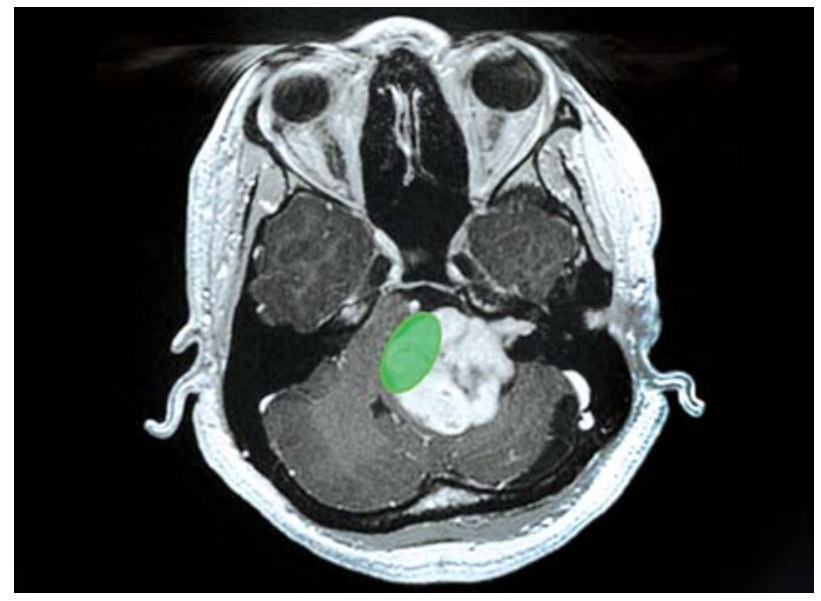

Fig. 1G: Demonstrates the brainstem portion of the tumor outlined in green on the same cut

\section{Internal Auditory Canal Portion}

While drilling the internal auditory canal (IAC) and removing the tumor from the fundus entails some work, it generally is a less risky maneuver in this surgery (Fig. $1 \mathrm{H}$ ). The anatomy of the facial nerve is relatively constant (anterior and superior to the tumor in the canal) in this region, and dense fibrous adhesions are uncommon, making this effort usually worthwhile. In hearing preservation cases, removing the IA C component can make the remnant a better radiosurgery target by increasing the distance from the cochlea and reducing the cochlear radiation dose, which probably improves hearing preservation. When applicable, decompressing the IAC might reduce the risk of delayed hearing loss caused by tumor remnants, by reducing the risk of intratumoral microhemorrhage and increase in IAC pressure that may cause hearing loss. ${ }^{15}$

\section{The Cisternal Portion}

The portion of tumor along the cisternal segment of the facial nerve (Fig. 1I) possibly poses the greatest challenge towards the goal of safe, complete tumor removal. The facial nerve is usually located somew here under the anterior surface of this tumor portion, however, the course can be highly variable, especially in large tumors, and in many cases, can make sharp bends even when the course seems cl ear, putting the facial nerve at risk. The tumor is frequently very adherent to the facial and cochlear nerves at the porus acousticus and the cisternal segment, and this is often the point in the operation when spontaneous facial nerve EM G activity is noted. Given that the tumor is typically a small, and possible devascularized remnant by this step in the operation, the author generally avoids heroic efforts to remove this portion, opting instead to follow the remnant with serial imaging and/or treating with radiosurgery shortly postoperatively or at growth of the fragment, depending on the volume of residual disease on postoperative imaging. Ideally, the

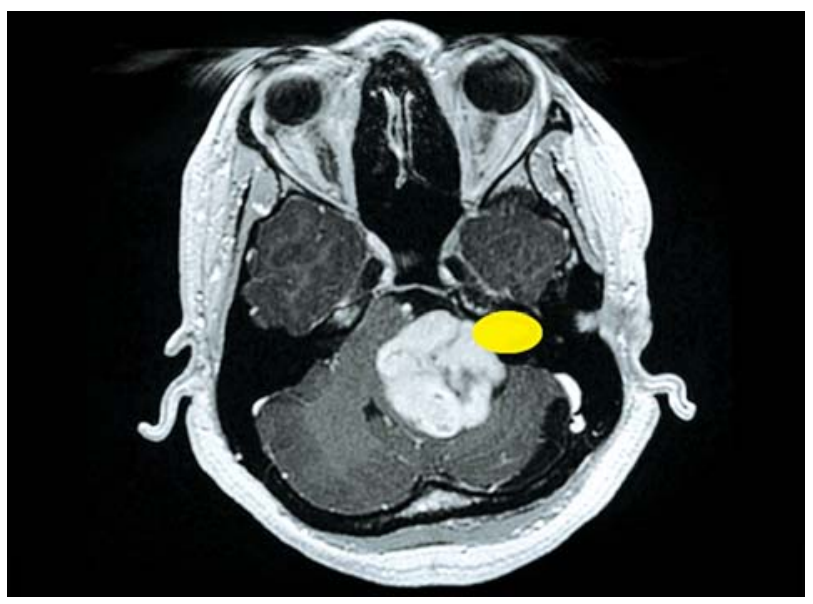

Fig. 1H: Demonstrates the IAC portion of the tumor outlined in yellow 


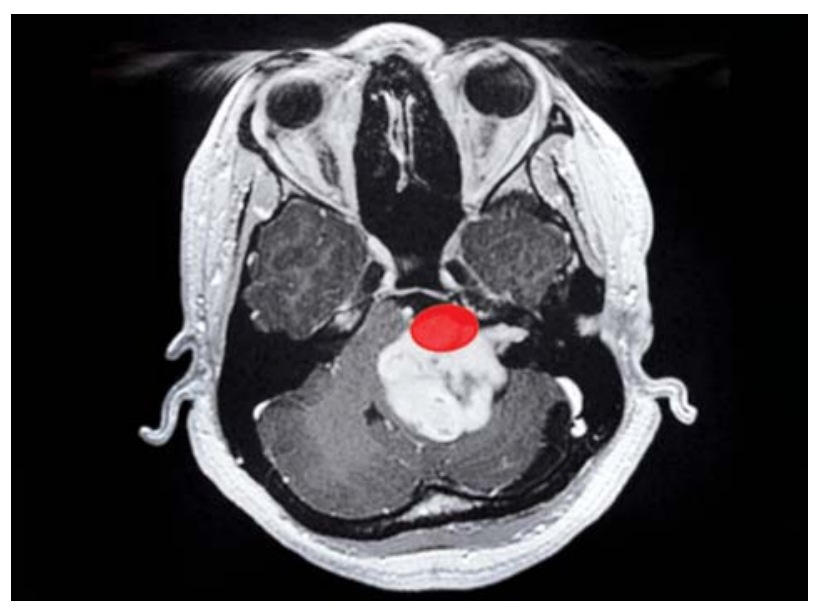

Fig. 1I: Demonstrates the cisternal portion of the tumor in red. The author suggests that this is probably the riskiest portion of the tumor to completely resect

cisternal segment of the facial nerve should not be visualized at all, making the risk of traction or devascularization injury minimal.

\section{Apical Segment}

This catch-all term describes tumor components (Figs 1] and K), only present in particularly large schwannomas, notably present at petrous apex and up near the anterior tentorial incisura. While tumor is not particular adherent to the cranial nerves here (trigeminal, abducens, and possibly the trochlear nerves) the relative worth of aggressively pursuing this portion should be considered in the general context of the rest of the resection.

\section{When to Administer Radiosurgery?}

There is little evidence to guide this decision. In many cases (over $80 \%$ at 10 years), the residual fragment will not grow during follow-up, ${ }^{11}$ and this suggests that surgical trauma and tumor devascularization to the tumor can drive many of these small fragments into growth arrest, and that immediate treatment is not al ways necessary. The growth rate and pattern of growth of residuals that do begin to regrow is not well-established, and little data specifically supports early radiosurgery over radiosurgery administered at the time of regrowth. A t present, the decision is probably best made in the context of the volume of residual disease, patients with large residual tumor volumes have less room to grow before they exceed radiosurgery volume, and thus early treatment seems more important to prevent the need for repeat surgery, than for small residual tumors, which probably can be watched.

\section{CONCLUSION}

While good surgical technique clearly can reduce the risk of facial nerve palsy in complete surgical resection
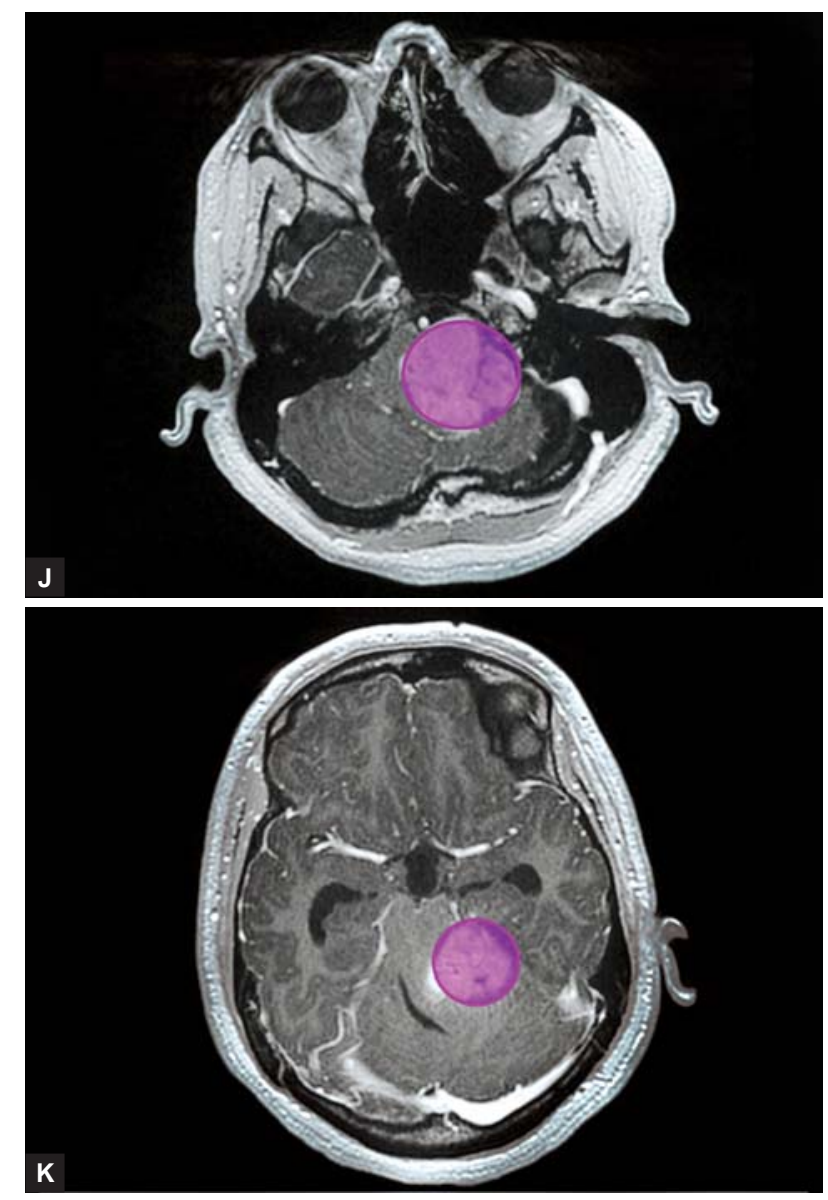

Figs $1 \mathrm{~J}$ and $\mathrm{K}$ : Demonstrate the tumor sections above and below; the IAC cut with the apical portions of tumor outlined in purple. In most cases, these can be removed safely, however, can be challenging in some cases

paradigms, even in the best hands it cannot eliminate this risk, especially with larger tumors. Radiosurgery generally can nearly eliminate this risk, and thus it seems reasonable to suggest that the role of surgery is to create a better radiosurgery target when necessary. The goal of elimination of facial nerve palsy in vestibular schwannoma management seems achievable with rational surgical management of these tumors, and methodical investigation of surgical strategies to validate and refine these techniques.

\section{REFERENCES}

1. Ballance. Some points in the surgery of the brain and its membranes. London: M ac M illian and C o. Ltd. 1907.

2. Cushing. Tumors of the nervus acusticus and the syndrome of the cerebellopontine angle. Philadel phia: London: WB Saunders 1917:296.

3. Sughrue ME, Y ang I, A randa D, K ane AJ, Parsa AT. Hearing preservation rates after microsurgical resection of vestibular schwannoma. J Clin Neurosci Sep 17(9):1126-29.

4. Sughrue ME, Y ang I, A randa D, et al. Beyond audiofacial morbidity after vestibular schwannoma surgery. J N eurosurg 27 Nov, 2009. 
5. Sughrue ME, Y ang I, Rutkowski MJ, A randa D, Parsa AT. Preservation of facial nerve function after resection of vestibular schwannoma. Br J Neurosurg Dec 24(6):666-71.

6. Sughrue ME, Y ang I, Han SJ, et al. N on-audiofacial morbidity after gamma knife surgery for vestibular schwannoma. Neurosurg Focus Dec 2009;27(6):E4.

7. Lunsford LD, Niranjan A, Flickinger JC, Maitz A, Kondziolka D. Radiosurgery of vestibular schwannomas: Summary of experience in 829 cases. J Neurosurg Jan 2005;102(Suppl):195-99.

8. $Y$ ang I, Sughrue ME, Han SJ, et al. Facial nerve preservation after vestibular schwannoma gamma knife radiosurgery. J Neurooncol M ay 2009;93(1):41-48.

9. Sughrue $M E, K$ ane $A$ J, K aur R, et al. A prospective study of hearing preservation in untreated vestibular schwannomas. J N eurosurg M ay 21.

10. Flickinger J C, K ondziolka D, N iranjan A, M aitz A, V oynov G, $L$ unsford $L D$. A coustic neuroma radiosurgery with marginal tumor doses of 12 to $13 \mathrm{~Gy}$. Int J Radiat O ncol Biol Phys 1 Sep 2004;60(1):225-30.

11. Sughrue ME, K aur R, Rutkowski MJ, et al. Extent of resection and the long-term durability of vestibular schwannoma surgery. J Neurosurg J an 21.
12. Sughrue ME, K aur R, Rutkowski MJ, et al. A critical evaluation of vestibular schwannoma surgery for patients younger than 40 years of age. N eurosurgery. Dec 67(6):1646-1653; Discussion 1653-44.

13. Rowe J, Grainger A, Walton L, Silcocks P, Radatz $M$, Kemeny $A$. Risk of malignancy after gamma knife stereotactic radiosurgery. N eurosurgery. J an 2007;60(1):60-65; Discussion 65-66.

14. Sughrue M E, Kaur R, K ane AJ, et al. The value of intraoperative facial nerve electromyography in predicting facial nerve function after vestibular schwannoma surgery. J Clin Neurosci; Jul 17(7):849-52.

15. Sughrue $M E, K$ aur $R, K$ ane $A$ J , et al. Intratumoral hemorrhage and fibrosis in vestibular schwannoma: A possible mechanism for hearing loss. J N eurosurg J un 18.

16. Sughrue $M E, Y$ ang $I, A$ randa $D$, et al. The natural history of untreated sporadic vestibular schwannomas: A comprehensive review of hearing outcomes. J N eurosurg 19 J un, 2009.

17. Sughrue ME, Y eung A H, Rutkowski MJ, C heung SW, Parsa AT. Molecular biology of familial and sporadic vestibular schwannomas: Implications for novel therapeutics. J N eurosurg 27 Nov, 2009.

18. Jian BJ, Sughrue ME, Kaur R, et al. Implications of cystic features in vestibular schwannomas of patients undergoing microsurgical resection. N eurosurgery 6 Jan. 